\title{
Subcortical Vascular Lesions Predict Functional Recovery After Rehabilitation in Patients with L-Dopa Refractory Parkinsonism
}

\author{
Fabio Guerini, MD, ${ }^{* \dagger}$ Giovanni B. Frisoni, MD ${ }^{\dagger \dagger}$ Catherine Bellwald, MD, ${ }^{* \dagger}$ Roberta Rossi, Psy, ${ }^{\dagger}$ \\ Giuseppe Bellelli, MD, ${ }^{*}$ and Marco Trabucchi, $M D^{\dagger \mathcal{S}}$
}

OBJECTIVES: To identify predictors of functional recovery after an intensive rehabilitation training in patients with gait disturbances and refractory parkinsonism.

DESIGN: Observational study.

SETTING: A hospital geriatric rehabilitation department (“Ancelle della Carità" hospital of Cremona).

PARTICIPANTS: Thirty-eight subjects (mean age \pm standard deviation of $78.9 \pm 6.5 ; 66 \%$ women) with gait disturbances and L-dopa refractory parkinsonism consecutively admitted to a rehabilitation unit within 6 months were recruited. Exclusion criteria were obvious musculoskeletal disorders (severe leg arthritis, hemiparesis, recent stroke), recent surgery, delirium, physical impairment from other identifiable causes, and missing computed tomography (CT) scan. All subjects received an intensive standardized rehabilitative program including conventional physical therapy and specific gait training.

MEASUREMENTS: The outcome measure of the rehabilitation training was the gain between admission and discharge on the Unified Parkinson Disease Rating Scale (delta-UPDRS). The following potential predictors were assessed using comprehensive geriatric assessment: physical health (Charlson Comorbidity Index, number of drugs), cognitive performance (Mini-Mental State Examination (MMSE)), functional status (Tinetti scale), depressive symptoms (Geriatric Depression Scale), nutritional status (serum albumin and body mass index), and subcortical cerebrovascular load (four classes of increasing severity based on diffuse leukoariosis, patchy lesions of the white matter, and lacunas on CT scan). Multivariate logistic regression with fixed adjustment for age, cognitive performance, and UPDRS on admission and stepwise selection of variables were used to identify independent predictors.

RESULTS: Patients were divided into two groups of equal size based on the delta-UPDRS (high and low functional recovery: delta-UPDRS $>8$ and $\leq 8$, respectively). Of all

From the *Rehabilitation Unit "Ancelle della Carità" Hospital, Cremona, Italy; ${ }^{\dagger}$ Geriatric Research Group, Brescia, Italy; ${ }^{\ddagger}$ Laboratory of Epidemiology and Neuroimaging, IRCCS S. Giovanni di Dio Fatabenefratelli, Brescia, Italy; and \&"Tor Vergata" University, Rome, Italy.

Address correspondence to Giuseppe Bellelli, MD, Head, Rehabilitation Unit, "Ancelle della Carità" Hospital, 26100, Cremona, Italy.

E-mail: bellelli-giuseppe@poliambulanza.it variables, only subcortical cerebrovascular load predicted functional recovery at discharge (odds ratio adjusted for age, MMSE, and UPDRS on admission $=2.3$, 95\% confidence interval $=1.0-5.1)$. The adjusted proportion of patients with high functional recovery decreased with increasing subcortical cerebrovascular load: $83 \%, 61 \%$, $44 \%$, and $27 \%$ (adjusted $P$ for trend $=0.047$ ).

CONCLUSION: Subcortical cerebrovascular load is a predicting factor of successful rehabilitation in patients with L-dopa refractory parkinsonism. J Am Geriatr Soc 52: 1-5, 2004.

Key words: parkinsonism; subcortical vascular lesions; functional recovery; rehabilitation.

$P$ arkinsonism is common in people aged 65 and older, and its prevalence increases markedly with age. ${ }^{1}$ In a study on 467 residents of East Boston, Massachusetts, 159 persons aged 65 and older were found to have parkinsonism. The overall prevalence estimates were $14.9 \%$ for people aged 65 to $74,29.5 \%$ for those aged 75 to 84 , and $52.4 \%$ for those aged 85 and older. In particular, gait disturbances, a key signs of parkinsonism, were found to be associated with twice the risk of death within a 9.2-year follow-up period. ${ }^{2}$ In another study on 15,034 patients in Ontario, ${ }^{3} 50.6 \%$ of subjects with parkinsonism died during the 6 -year follow-up in comparison with $29.1 \%$ of control subjects.

Only a portion of all patients with parkinsonism have idiopathic Parkinson's disease (PD), whereas the rest have other forms, generally poorly responsive or altogether refractory to L-dopa or other dopaminergic therapy. ${ }^{1,3}$ Nevertheless, despite an overall positive effect of physical rehabilitation on gait disturbances in patients with L-dopa refractory parkinsonism, the variability of the response is large. ${ }^{4}$ Therefore, the identification of predictors of response would be of great clinical value. Some authors have suggested that age, cognitive impairment, delirium, mood, comorbidity, malnutrition, and functional status might predict the success of rehabilitation, ${ }^{5-8}$ but increasing attention is being paid to subcortical cerebrovascular 
disease; several studies have shown the high prevalence and the association of these lesions with gait disturbances. ${ }^{9-11}$

In this framework, the aim of the current study was to identify independent predictors of functional recovery after intensive rehabilitation training in patients with gait disturbances and refractory parkinsonism.

\section{METHODS}

\section{Subjects}

Patients affected by L-dopa refractory parkinsonism were selected from among all first and consecutive admissions to the rehabilitation unit of "Ancelle della Carità" Hospital, Cremona, Italy, a 30-bed rehabilitation unit, between October 2001 and March 2002. The total turnover of the unit is about 380 patients per year.

L-dopa refractory parkinsonism was defined as the presence of at least two of the four cardinal signs: rest tremor, rigidity, bradykinesia, and gait disturbances due to loss of postural reflexes. ${ }^{12}$ The refractoriness to L-dopa was defined as an absent or minimal clinical response to a 3-consecutive-day pharmacological trial of carbidopalevodopa (up to 25-250 mg of Sinemet three times daily). ${ }^{13}$ Exclusion criteria included obvious musculoskeletal disorders (e.g., severe leg arthritis, hemiparesis, recent stroke), recent surgery, delirium, physical impairment from other identifiable causes, and missing computed tomography (CT) scan.

\section{Comprehensive Geriatric Assessment}

All patients had a comprehensive geriatric assessment performed by a geriatrician (GB) on admission, including:

demographics: age, sex, education, and living condition cognitive performance, using the Mini-Mental State Examination (MMSE) $)^{14}$

depressive symptoms, using the 15 -item Geriatric Depression Scale ${ }^{15}$

functional status, using the Tinetti scale ${ }^{16}$ and the Barthel Index ${ }^{17}$

physical health, using the Charlson Comorbidity Index ${ }^{18}$ and number of drugs

malnutrition, determined from serum albumin and body mass index ${ }^{19}$

The outcome measure was a sum score of the motor and activities of daily living (ADL) subscales of the Unified Parkinson's Disease Rating Scale (UPDRS). ${ }^{20}$ The UPDRS is the most widely used instrument for rating symptom severity in clinical studies of PD. The ADL subscale is based on historical information, and the motor subscale is based on a structured physical examination. The sum score of the two subscales ranges from 0 to 108 , higher scores indicating greater impairment and vice versa. In all cases, a trained physiotherapist blind to the aim of the study administered the UPDRS subscales at admission and discharge. A delta-UPDRS score was calculated as the difference between scores on admission and discharge. Moreover, a sum score assessing executive control functions was computed from the following items: writing, dressing, grooming, and tool use.

\section{Rehabilitation Training}

All subjects received an intensive standardized rehabilitative program including conventional physical therapy $(40$ minutes in the morning) and specific training (40 minutes in the afternoon). The global amount of the rehabilitative program per patient was 80 minutes per day, 6 days per week, for 3 consecutive weeks.

The treatment areas used for conventional physiotherapy included strengthening and range-of-motion exercises, musculoskeletal control, trunk and upper extremity positioning, transfer training, postural and gait training, functional and self-care retraining, and adaptive equipment training. Each session consisted of a 40-minute phase of two sets of 12 repetitions of nine exercises: leg extension, leg curl, step-up, heel raise, chest fly, upright row, military press, biceps curl, and pelvic tilt.

The specific treatment was based on treadmill training cycle at an intensity equivalent to $50 \%$ to $70 \%$ of the participants' heart reserve, as determined from their screening exercise treadmill test. At the start of each session of the rehabilitation training, the physiotherapist explained the nature and type of the exercises. When patients required rest, a brief interruption (no longer than 1 minute) was allowed. Each session of physiotherapy began with a 15minute warm-up and ended with 10-minute cool-down.

\section{Classification of Cerebrovascular Severity}

Cerebrovascular severity was assessed on CT films with a rating scale for subcortical cerebrovascular disease. ${ }^{21}$ Three types of lesions (leukoariosis, fuzzy or patchy lesions, and lacunas) were rated separately for the right and left hemispheres in a number of brain regions and summed to give a single measure of subcortical vascular load (SVL).

\section{Leukoariosis}

The degree of hypodensity of the white matter was quantified separately in three regions (frontal, parietal and occipital). A score of 0 indicated no hypodensity, 1 questionable hypodensity (might be regarded as "normal for age"), 2 definite hypodensity but confined to the periventricular area or not reaching the cortex, and 3 marked hypodensity reaching the cortex or extending into the white matter of the gyral digitations. A sum score of leukoariosis, ranging between 0 and 18, was computed and categorized into three classes of severity: score of 0 to $9=$ Class A, 10 to $16=$ Class B, and 17 and higher $=$ Class $\mathrm{C}$.

\section{Patchy Hypodensities}

The presence of focal areas of hypodensity with indistinct boundaries was separately assessed in seven regions: frontal, temporal, parietal, and occipital lobes; basal ganglia; internal capsule; external capsule; and cerebellum. The total number of patchy hypodensities was recorded and categorized into three levels of increasing severity: 0 lesions $=$ Class A, 1 to $3=$ Class B, and 4 and more $=$ Class $\mathrm{C}$.

\section{Lacunas}

These were assessed in the same seven regions as the patchy hypodensities and were described as well-defined areas of marked and homogeneous hypodensity with a well-defined 
and regular contour measuring generally from 2 to $10 \mathrm{~mm}$ across. The total number of lacunas was recorded and categorized into three levels of increasing severity: 0 lesions $=$ Class A, 1 to $2=$ Class $\mathrm{B}$, and 3 and more $=$ Class $\mathrm{C}$.

To obtain a global measure of subcortical cerebrovascular disease, an ordinal (1-4) four-level variable was created (SVL), with 1 indicating absent or very mild (Class $\mathrm{B}$ leukoariosis or lower and Class A ptachy lesions and lacunas), 2 as mild (Class $\mathrm{C}$ leukoariosis or Class B patchy and Class A lacunas), 3 as moderate (Class B patchy and Class A lacunas irrespective of class of leukoariosis), and 4 as severe disease (Class B or higher patchy lesions and Class $\mathrm{B}$ or higher lacunas irrespective of leukoariosis).

\section{Statistical Analysis}

Independent predictors of functional recovery were tested in a logistic regression model with high/low functional recovery as the dependent variable; fixed adjustment for age, MMSE, and UPDRS at baseline; and a stepwise selection method to identify independent predictors $(P<.05$ of the score statistic for variable entry and $P>.10$ of the conditional statistics for variable removal). The findings were confirmed in a homologous linear regression model with the delta-UPDRS as a continuous variable. Adjusted prevalence of high gain in classes of vascular score were computed based on adjusted odds ratios computed in a multivariate logistic regression model with age, MMSE, and UPDRS on admission as covariates and using level 1 of SVL as reference group.

\section{RESULTS}

Table 1 shows the clinical characteristics of the patients stratified into two groups of equal size based on the delta-
UPDRS (high functional recovery, low functional recovery). Patients had similar demographic, cognitive, affective, somatic, and functional characteristics on admission. The sum score (mean \pm standard deviation of UPDRS items assessing executive control functions was not different in the two groups of high and low functional recovery $(6.3 \pm 3.7$ vs $7.2 \pm 3.5, P=.448))$. Although both groups had significant functional improvement at discharge, the group with high functional recovery had gained 9.1 UPDRS points more than the group with low functional recovery.

The mean number of lacunas $(0.79 \pm 1.75$ vs $0.21 \pm 0.53)$, patchy lesions $(4.1 \pm 2.5$ vs $3.1 \pm 3.4)$, and leukoariosis $(12.9 \pm 5.3$ vs7.7 \pm 5.1$)$ were more severe in the low than high functional recovery group, although only leukoariosis attained statistical significance $(P=.003)$. Cortical strokes on CT scan were not significantly different in the two groups $(5.3 \%$ vs $15.8 \%$ in those with high and low functional recovery, $P=.612$ on $\chi^{2}$ test), but SVL was significantly different $\left(P=.032\right.$ on $\chi^{2}$ test).

Table 2 shows that the only predictor of functional recovery at discharge was SVL (adjusted test for trend: $P=.047$ ). Body mass index was the variable with the next highest predictive power, but its $P$-value was far from significance $(P=.250$ of the score statistics for variable entry). Confirmatory analysis with linear regression showed that, after the effects of age $(P=.858)$, MMSE $(P=.271)$, and UPDRS on admission $(P=.90)$ were parceled out, SVL was the only independent predictor (regression coefficient $=-0.20,95 \%$ confidence interval $=-0.34$ to $-0.06, P=.011$ ).

Figure 1 shows that the distribution of the two groups (low and high functional recovery) in four levels of SVL adjusted for age, MMSE, and UPDRS was clearly skewed toward higher values in the low functional recovery group, arguing for a dose-effect relationship between SVL and

Table 1. Clinical and Functional Characteristics of 38 Patients with L-Dopa Refractory Parkinsonism Treated with an Intensive Rehabilitation Training Stratified by Functional Recovery (Difference between Discharge and Admission Unified Parkinson Disease Rating Scale UPDRs)

\begin{tabular}{|c|c|c|c|}
\hline \multirow[b]{2}{*}{ Characteristic } & $\begin{array}{l}\text { High Functional } \\
\text { Recovery }\left(n=19^{*}\right)\end{array}$ & $\begin{array}{c}\text { Low Functional } \\
\text { Recovery }\left(n=19^{\dagger}\right)\end{array}$ & \multirow[b]{2}{*}{ P-value ${ }^{\ddagger}$} \\
\hline & \multicolumn{2}{|c|}{ Mean \pm Standard Deviation (Range) } & \\
\hline Age & $78.8 \pm 6.6(64-89)$ & $78.9 \pm 6.5(67-91)$ & .838 \\
\hline Mini-Mental State Examination (range 0-30) & $24.5 \pm 3.5(17-28)$ & $21.9 \pm 5.3(10-30)$ & .117 \\
\hline Geriatric Depression Scale (range 0-15) & $5.1 \pm 3.3(1-13)$ & $6.3 \pm 2.9(2-13)$ & .171 \\
\hline Body mass index, $\mathrm{kg} / \mathrm{m}^{2}$ & $23.7 \pm 4.8(14.3-32.5)$ & $22.1 \pm 6.0(12.2-28.7)$ & .726 \\
\hline Drugs, $\mathrm{n}$ & $4.7 \pm 1.1(3-8)$ & $5.1 \pm 1.3(3-7)$ & .421 \\
\hline Duration of gait symptoms, years & $3.6 \pm 3.1(1-10)$ & $3.7 \pm 4.1(0-15)$ & .777 \\
\hline Barthel index (0-100) & $64.7 \pm 28.8(10-100)$ & $71.2 \pm 24.3(10-100)$ & .438 \\
\hline Tinetti scale admission (0-28) & $13.6 \pm 6.5(2-24)$ & $13.0 \pm 5.6(6-26)$ & .549 \\
\hline
\end{tabular}


Table 2. Predictors of Poor Functional Recovery Following Intensive Rehabilitation Training in 38 Patients with LDopa-Refractory Parkinsonism

Odds Ratio $(95 \%$

Predictor $\quad$ Confidence Interval) $P$-value

\begin{tabular}{lll}
\hline Fixed adjustment (enter) & & \\
$\quad$ Age & $1.00(0.9-1.1)$ & .987 \\
$\quad$ Mini-Mental State Examination & $0.88(0.7-1.1)$ & .165 \\
$\quad$ UPDRS admission & $1.01(1.0-1.1)$ & .769 \\
$\quad \begin{array}{l}\text { Stepwise selection } \\
\quad \text { Subcortical vascular load }\end{array}$ & $2.3(1.0-5.1)$ & .047 \\
\hline
\end{tabular}

Note: Coefficients were computed in a multiple logistic regression model with fixed adjustment for age, Mini-Mental State Examination, and UPDRS on admission and stepwise selection of all other predictors. Sex, Geriatric Depression Scale, Charlson index, serum albumin, body mass index, drugs, duration of gait symptoms, and Tinetti scale admission were not selected to enter the model. UPDRS $=$ Unified Parkinson Disease Rating Scale (subscales 2 and 3).

functional recovery. The adjusted frequency of poor functional recovery was $17 \%, 39 \%, 56 \%$, and $73 \%$ in classes of increasing SVL.

\section{DISCUSSION}

This study shows that SVL may influence functional recovery after intensive rehabilitation training in elderly patients refractory to L-dopa parkinsonism.

Several experiments in animals have recently shown that strokes are followed by new cortical representations of the affected limbs that may be modified by intensive repetition of single and combined movements. ${ }^{22,23}$ Tactile tasks and sensorial stimuli can promote the remodeling and reactivation of cortical areas involved in motor processing. ${ }^{22}$ A similar reorganization has been reported in patients who have recovered from lacunar infarction, but only if they are engaged in vigorous physical training. ${ }^{23}$

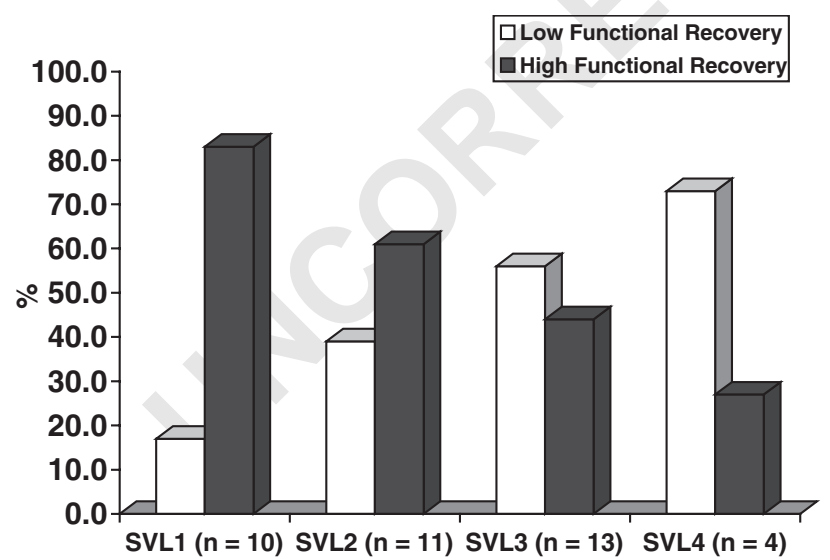

Figure 1. Adjusted frequency of high and low functional recovery in classes of increasing subcortical vascular load (SVL). Higher SVL number denotes SVL of greater severity (see Methods). Adjustment was made for age, Mini-Mental State Examination score, and Unified Parkinson Disease Rating Scale on admission. $P$ denotes significance on test for trend computed in a multiple logistic regression model (Table 2).
This remodeling continues long after the damage, suggesting that neuroplastic changes may also be present in the chronic stage. ${ }^{24,25}$

According to this view, intensive cycles of physical rehabilitation are needed to obtain functional improvement in gait disturbances secondary to neuronal brain damage. However, the findings of the current study showed that comparable intensity of rehabilitative training did not allow homogeneous functional outcomes in patients. It was found that subjects with lower functional recovery differed from the others by having a greater amount of SVL. This corresponds with previous reports indicating that anatomic location and entity of subcortical cerebrovascular disease can strongly influence functional improvement of patients with parkinsonian syndromes; ${ }^{26,27}$ the subcortical regions are critical in integrating the sensory and motor processing underlying the complex spectrum of behaviors required for mobility ${ }^{22}$ Maintenance of correct posture and kinematic tasks requires ongoing synthesis of visual, proprioceptive, somatosensory, vestibular, and motor processing in real time. Lesions affecting the integrity of any neuronal circuit or the connections between circuits of the network might decrease the efficiency or altogether disable this association processing, causing a sensorimotor "disconnection syndrome."

It is remarkable that a dose-effect relationship between functional recovery and SVL, suggesting that the greater the subcortical vascular damage the greater the deterioration of neuronal tracts involved in control of gait and balance, was observed.

These findings could have implications for the rehabilitation of patients with refractory parkinsonism, because they indicate that patients with greater SVL might need more-intensive rehabilitation training. It is conceivable that, in these patients, the simultaneous stimulation of motor and somatosensory neuronal networks could facilitate the reactivation of neuroplastic processes and allow better functional outcomes.

Some caveats must be highlighted. First, CT rather than the state-of-the-art magnetic resonance image (MRI) was used to assess SVL. Nevertheless, although changes as small as a few $\mathrm{mm}$ can be appreciated with MRI with sharp hyperintense signals in adequate sequences (T2 and FLAIR), CT has greater specificity. ${ }^{28}$ Moreover, studies have shown a good correlation between CT-based and MRI-based detection of subcortical lesions. ${ }^{29}$ Second, although all patients received a 3-day trial of L-dopa, it cannot be excluded that some patients with idiopathic PD were included. Nevertheless, it is likely that PD patients were homogeneously distributed between the two groups. Last, it might be hypothesized that executive control function rather than SVL is the major source of variance in functional outcome; executive control function is strongly associated with both subcortical vasculopathy and functional outcomes. However, the UPDRS items assessing executive control function were not different in those with high and low functional recovery. ${ }^{30}$

In conclusion, the results of this study suggest that SVL is a predicting factor of the success of rehabilitation in patients with L-dopa refractory parkinsonism. Future studies will need to evaluate whether more-intensive and differentiated rehabilitation training could be effective for 
patients with L-dopa refractory parkinsonism and severe subcortical cerebrovascular disease.

\section{ACKNOWLEDGMENTS}

Sincere appreciation is due to Dr. Elena Lucchi and Dr. Francesca Magnifico for their support in cognitive assessment and to Mr. Daniele de Palma for the support in rehabilitative training.

\section{REFERENCES}

1. De Rijk MC, Tzourio C, Breteler MMB et al. Prevalence of parkinsonism and Parkinson's disease in Europe: The EUROPARKINSON Collaborative Study. J Neurol Neurosurg Psychiatry 1997;62:10-15.

2. Bennet DA, Beckett LA, Murray AM et al. Prevalence of parkinsonian signs and associated mortality in a community population of older people. N Engl J Med 1996;334:71-76.

3. Guttman M, Slaughter PM, Theriault M et al. Parkinsonism in Ontario. Increased mortality compared with controls in a large cohort study. Neurology 2001;57:2278-2282.

4. Liston R, Mickelborough J, Harris B et al. Conventional physiotherapy and treadmill re-training for higher level gait disturbances in cerebrovascular disease. Age Ageing 2000;29:311-318.

5. Leveille SG, Guralnik JM, Ferrucci L et al. Aging successfully until death in old age: Opportunities for increasing active life expectancy. Am J Epidemiol 1999;149:654-664.

6. Landi F, Bernabei R, Russo A et al. Predictors of rehabilitation outcomes in frail patients treated in a geriatric hospital. J Am Geriatr Soc 2002;50:679684.

7. Barbisoni P, Bertozzi B, Franzoni S et al. Mood improvement in elderly women after in-hospital physical rehabilitation. Arch Phys Med Rehabil 1996;77:346349.

8. Patrick L, Knoefel F, Gaskowsky P et al. Medical comorbidity and rehabilitation efficiency in geriatric inpatients. J Am Geriatr Soc 2001;49: 1471-1477.

9. Whitman GT, Tang T, Lin A et al. A prospective study of cerebral white matter abnormalities in older people with gait dysfunction. Neurology 2001;57:990994.

10. Benson RR, Guttmann CRG, Wie X et al. Older people with impaired mobility have specific loci of periventricular abnormality on MRI. Neurology 2002;58:48-55.

11. Winikates J, Jankovic J. Clinical correlates of vascular parkinsonism. Arch Neurol 1999;56:98-102.

12. Yamanouchi $H$, Nagura $H$. Neurological signs and frontal white matter lesions in vascular parkinsonism. A clinicopathologic study. Stroke 1997;28:965-969.
13. Zijlmans JCM, Thijssen HOM, Vogels OJM et al. MRI in patients with suspected vascular parkinsonism. Neurology 1995;45:2183-2188.

14. Folstein MF, Folstein S, McHugh PR. 'Mini-mental-state.' A practical method for grading cognitive state of patients for the clinician. J Psychiatr Res 1975;12:189-198

15. Sheikh JI, Yesavage JA. Geriatric Depression Scale (GDS). Recent Evidence and Development of a Shorter Version. Clinical Gerontology: A Guide to Assessment and Intervention. New York: The Harworth Press, 1986, pp 165173.

16. Tinetti ME. Performance-oriented assessment of mobility problems in elderly patients. J Am Geriatr Soc 1986;34:119-126.

17. Mahoney FI, Barthel DW. Functional evaluation. The Barthel Index. Md State Med J 1965;14:61-65.

18. Charlson ME, Pompei P, Ales KL et al. A new method of classifying prognostic comorbidity in longitudinal studies: Development and validation. J Chronic Dis 1987;40:373-383.

19. Matthews LE. Using anthropometric parameters to evaluate nutritional status. J Nutr Elderly 1986;5:67-71.

20. Fahn S, Elton R. Unified Parkinson's Disease Rating Scale. In: Fahn S, Marsden C, Goldstein M et al., eds. Recent Developments in Parkinson's Disease, Vol. 2. Florham Park, NJ: MacMillan Health Care Information, 1987, pp 153-163.

21. Geroldi C, Galluzzi S, Bresciani L et al. A hierarchical scale to assess brain vascularity on $\mathrm{CT}$ films in dementing and non-dementing cognitive impairment. J Neurol 2000;247(Suppl 3):III/31.

22. Nadeau SE. A paradigm shift in neurorehabilitation. Lancet Neurol 2002;1:126-130.

23. Gladstone DJ, Black SE, Hakim AM et al. Toward wisdom from failure. Lesson from neuroprotective stroke trials and new therapeutic directions. Stroke 2002;33:2123-2136

24. Jenkins WM, Merzenich MM, Ochs MT et al. Functional reorganization of primary somatosensory cortex in adult owl monkeys after behaviorally controlled tactile stimulation. J Neurophysiol 1990;63:82-104.

25. Liepert J, Bauder H, Wolfang HR et al. Treatment-induced cortical reorganization after stroke in humans. Stroke 2000;31:1210-1216.

26. Miyai I, Blau AD, Reding MJ et al. Patients with stroke confined to basal ganglia have diminished response to rehabilitation efforts. Neurology 1997;48:95-101.

27. Dromerick A, Reding MJ. Functional outcome for patients with hemiparesis, hemihyperesthesia, and hemianopsia. Stroke 1995;26:2023-2026.

28. Lopez OL, Becker JT, Jungreis CA et al. Computed tomography — but not magnetic resonance imaging - identified periventricular white matter lesions predict symptomatic cerebrovascular disease in probable Alzheimer's disease. Arch Neurol 1995;52:659-664.

29. Wahlund LO, Barkhof F, Fazekas F et al. A new rating scale for age-related white matter changes applicable to MRI and CT. Stroke 2001;32:1318-1322.

30. Bellelli G, Lucchi E, Cipriani G et al. Executive dysfunction and depressive symptoms in cerebrovascular disease. J Neurol Neurosurg Psychiatry 2002;73:462. 


\section{Author Query Form}

Journal JGS

\section{Article $\quad 52064$}

1. Disk Usage :-

$\square$ Disk not provided

$\square$ Disk used

$\square \quad$ Disk not used

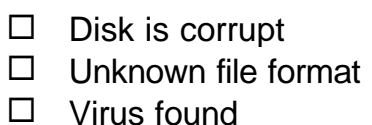

$\square \quad$ Additional Work done:

2. Queries :-

While preparing this paper/manuscript for typesetting, the following queries have arisen.

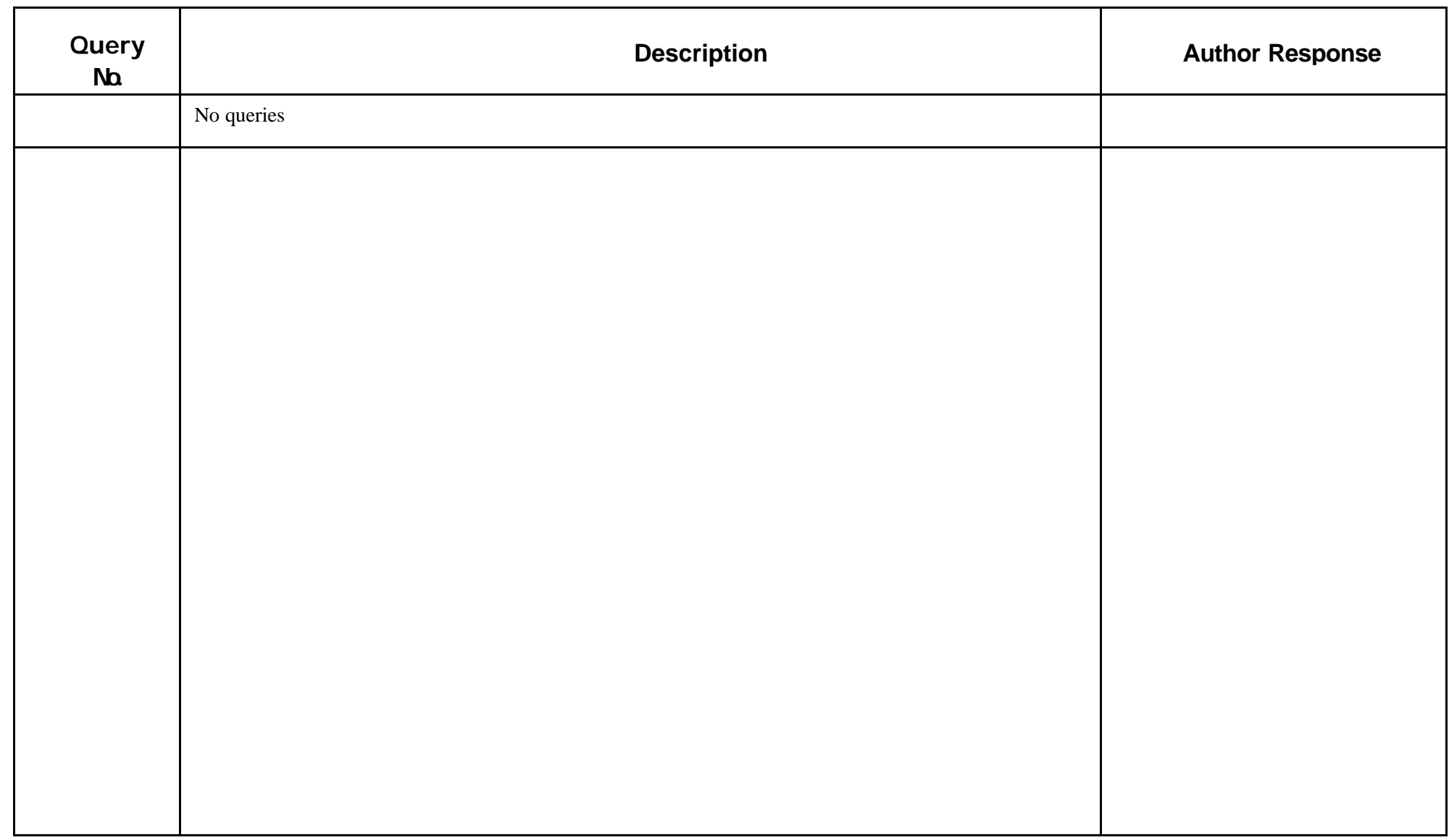

